\title{
Maneuvering Target Tracking Based on EBPSK Modulated JRC System
}

\author{
Yu Yao, Huanglong Teng, and Lenan $\mathrm{Wu}$
}

\begin{abstract}
Aiming at the existing problems of current algorithm for maneuvering target tracking, the paper proposed a new scheme that uses a joint radar-communication (JRC) system with Kalman filters to accurately tracking the target with the generalized likelihood ratio test (GLRT) theory. First, the tracking model of extended binary phase shift keying (EBPSK) modulated JRC system is introduced. Also, generalized likelihood ratio (GLR) and marginalized likelihood ratio (MLR) algorithm were given. Moreover, an adaptive maneuvering target tracking algorithm using interacting multiple model (IMM) technique is combined with Kalman filters to adapt the target maneuver. In addition to theoretical considerations, the EBPSK modulated JRC system based on the proposed algorithms on maneuvering target tracking problem are validated and evaluated by computer simulations.
\end{abstract}

Index Terms - Extended binary phase shift keying (EBPSK), interacting multiple model (IMM), Kalman filter, maneuvering target tracking, joint radar-communication.

\section{INTRODUCTION}

The extended binary phase shift keying (EBPSK) modulated joint radar-communications (JRC) system in the literature [1]-[4] showed excellent constant false alarm rate (CFAR) target detection performance when performing radar measurements for uniform motion and maneuvering targets. EBPSK modulation technique [5], [6] can achieve a very high spectrum efficiency without excessively penalizing the power efficiency. In addition to the high range resolution, the system is stable and offers reliable target detection ability in long distance [7], [8]. A simple amplitude threshold detector in the JRC system can demodulate the EBPSK modulated pulse echos, resulting in a simple receiver structure [9].

Maneuvering target tracking problem has attracted substantial interest, which is very meaningful for modern radars [10]-[13]. The generalized likelihood ratio (GLR) and marginalized likelihood ratio (MLR) method are proposed for maneuvering target tracking. The MLR test appears to be more efficient than the GLR test assuming more prior information [14]. In this paper, Aiming at the existing problems of current algorithm for maneuvering target tracking, a model adaptive maneuvering target tracking algorithm using IMM based Kalman filters is proposed.

Manuscript received June 24, 2015; revised December 29, 2015. This work was supported by the national Natural Science Foundation of China (6504000089)

$\mathrm{Yu}$ Yao and Lenan $\mathrm{Wu}$ are with the School of Information Science and Engineering, Southeast University, Nanjing, 210000 China (e-mail: shel18696@hotmail.com,wuln@seu.edu.com).

Huanglong Teng is with China Electronics Technology Group Corporation NO.7 Research Institute, Guangzhou, 510000 China (e-mail: tenghuanglong@163.com).
Weighted least square estimation for target location and Kalman filtering for target tracking based on one or more different models(maneuver model, the different parameter of maneuver model) are used in EBPSK modulated JRC system. The proposed algorithms offer good tracking accuracy and model adaptation as illustrated in the computer simulations.

The rest of this paper is organized as follows: Section II introduces the tracking model of EBPSK modulated JRC system. Section III describes generalized likelihood ratio algorithms. Section IV illustrates IMM based kalman filters. Some indicative simulation results and performance analysis are presented in Section V. And finally, Section VI gives the conclusion of the paper.

\section{THE MOdEL OF EBPSK MOdULATED JRC SYSTEM}

The EBPSK modulated JRC system model for tracking and estimation of maneuvering target in AWGN (Additive-White Gaussian Noise) is shown in Fig. 1.

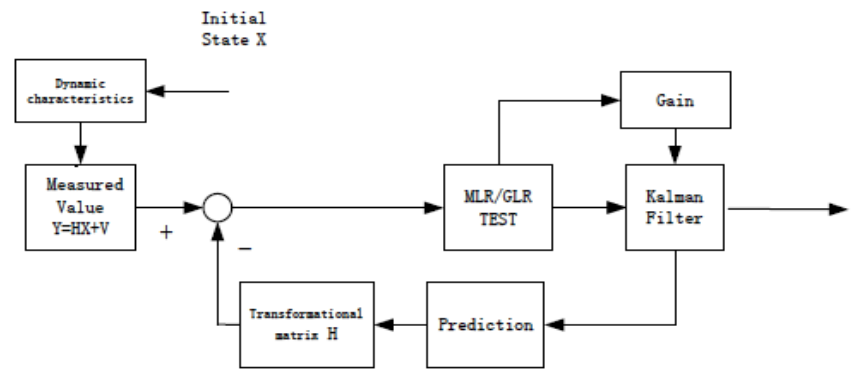

Fig. 1. The tracking model of EBPSK modulated JRC system

Let $u$ be the input that is responsible for maneuver and let $n$ be the maneuver onset time so that $u_{k}=0$ for $k<n$ and $u_{k} \neq 0$ for $k \geq n$ over the time window $[k-s, k)$. Consider the following maneuver hypotheses in terms of input value:

$$
\begin{aligned}
& H_{0}: u_{k}=0 \quad \text { for all } k \in[k-s, k) \\
& H_{1}(u, n): u_{n}=u_{n+1}=\ldots u_{k-1}=u \neq 0 \quad \text { for some } n \in[k-s, k)
\end{aligned}
$$

where the input level $u$ and the maneuver onset time $n$ are unknown. The likelihood ratio of $H_{1}$ vs $H_{0}$ with given $u$ and $n$ is:

$$
\Lambda(u, n)=\frac{f\left(z_{s}^{k} \mid H_{1}(u, n)\right)}{f\left(z_{s}^{k} \mid H_{0}\right)}
$$

where $z_{s}^{k}$ stands for set of measurements $\left\{z_{s}, \ldots, z_{k}\right\}$. A principle widely used is to replace the unknown likelihood functions by their maxima over the unknown parameters $(u, n)$. That is, replace $\quad f\left(z_{s}^{k} \mid H_{1}(u, n)\right) \quad$ with 
$f\left(z_{s}^{k} \mid H_{1}(\hat{u}, \hat{n})\right)=\max _{(u, n)} f\left(z_{s}^{k} \mid H_{1}(u, n)\right)$. Denote by $J(u, n)=\log \Lambda(u, n)$ the $\log$-likelihood ratio. The joint maximum likelihood estimate $(\hat{u}, \hat{n})$ is found in two steps as follows:

1) Find $\hat{u}(n)=\arg \max _{u} J(u, n)$ as the input estimate given onset time $n$ and then

$$
(\hat{u}, \hat{n})=\arg \max _{[\hat{u}(n), n]} J(\hat{u}(n), n)
$$

The main reason for this two-step approach is the ease at finding $\hat{u}(n)$.

2) The GLR maneuver detector declares detection of a maneuver if the generalized log-likelihood ratio:

$$
J(\hat{u}, \hat{n})=\log \frac{f\left(z_{s}^{k} \mid H_{1}(\hat{u}, \hat{n})\right)}{f\left(z_{s}^{k} \mid H_{0}\right)}
$$

exceeds a properly chosen threshold. Under the linear-Gaussian assumption, $\hat{u}(n)=\sum(n) e(n)$ is easily obtainable (the least-squares estimate of $u$ given $n$ ) where $\sum(n)=M S E[\hat{u}(n)]$ is the mean-spuare error matrix of $\hat{u}(n)$. It can be easily verified that:

$$
J[\hat{u}(n), n]=-\frac{1}{2} \sum_{k}\left(\tilde{z}_{k}^{*}\right)^{\prime} S_{k}^{-1} \tilde{z}_{k}^{*}+\frac{1}{2} \Delta J[\hat{u}(n), n]
$$

where $\tilde{z}_{k}^{*}$ is the residual at time $k$ under $H_{0}$ and $S_{k}=\operatorname{cov}\left(\tilde{z}_{k}^{*}\right)$.

The marginalized likelihood ratio (MLR) method appears to be more efficient than the GLR test assuming more prior information. Its basic idea is to obtain the marginal ML estimate $\hat{n}$ that has the maximum likelihood for an average $u$, rather than using the joint $\operatorname{MLE}(\hat{u}, \hat{n})$. In essence, MLR test checks the ratio of average likelihoods, as opposed to the ratio of most probable likelihoods in the GLR test. The hypotheis testing problem is formulated with respect to the marginalized log-likelihood ratio (MLR).

$$
J(n)=\log \frac{f\left(z_{s}^{k} \mid H_{1}(n)\right)}{f\left(z_{s}^{k} \mid H_{0}\right)}=\log \frac{E\left[f\left(z_{s}^{k} \mid H_{1}(u, n)\right)\right]}{f\left(z_{s}^{k} \mid H_{0}\right)}(6)
$$

where

$$
f\left(z_{s}^{k} \mid H_{1}(n)\right)=E\left[f\left(z_{s}^{k} \mid H_{1}(u, n)\right)\right]=\int f\left(z_{s}^{k} \mid H_{1}(u, n)\right) f(u) d u
$$

The test is $\max _{n} J(n)>0$. The input $u$ in the formulation is considered as a random variable, in contrast to the GLR method where it is assumed a deterministic constant. The input level is eliminated by averaging over all possible levels. Clearly the crucial problem of threshold determination of the GLR test is circumvented in the MLR formulation. Under some condition and with a special choice of the GLR threshold, both tests coincide. The MLR test is also more robust than the GLR test to unknown noise levels.

\section{GENERALIZED LiKELIHOOD RATIO ALGORITHMS}

In this algorithm, input estimates $\hat{\boldsymbol{u}}_{k}(n)$ for all possible maneuver onset time $n$ are computed and the one that maximizes the $\log$-likelihood $\log f\left(\bar{z}_{k}^{*} \mid \hat{u}, n\right)$ is taken to be the input estimate and the corresponding $n$ as the onset time estimate $\hat{n}$. The maneuver detection in this algorithm is done based on the generalized likelihood ratio (GLR) test, hence the name GLR algorithm. Specifically, this algorithm for MTT consists of the following:

1) Input estimation. For each $n=k-s, \ldots, k-1$, obtain the MLE $\quad \hat{u}_{k}(n)=\operatorname{argmax}_{u} \log f\left(\bar{z}_{k}^{*} \mid \hat{u}, n\right) \quad$, Then $\hat{u}_{k}(\hat{n})=\arg \max _{k-s \leq n<k} \log f\left(\bar{z}_{k}^{*} \mid \hat{u}_{k}(n), n\right)$.

2) Onset time estimation. Obtain the MLE $\hat{n}=\arg \max _{k-s \leq n<k} \log f\left(\bar{z}_{k}^{*} \mid \hat{u}_{k}(n), n\right)=\arg \max _{k-s \leq n<k} \Delta J_{k}\left[\hat{u}_{k}(n), n\right]$ which is given by the linear-Gaussian assumption.

3) Maneuver detection. A maneuver is declared if $\hat{u}_{k}(\hat{n})^{\prime}\left[\sum_{k}(\hat{n})\right]^{-1} \hat{u}_{k}(\hat{n})>\lambda$.

4) State estimate correction. Use $\hat{x}_{k \mid k-1}=\hat{x}_{k \mid k-1}^{*}+\hat{\mathbf{G}}_{k} \hat{\mathbf{u}}_{k}$ and $P_{k \mid k-1}=\operatorname{MSE}\left(\hat{x}_{k \mid k-1}\right) \approx P_{k \mid k-1}^{*}+\hat{\mathbf{G}}_{k} \sum_{k} \hat{\mathbf{G}}_{k}^{\prime} \quad$ if $\quad$ a maneuver is declared.

\section{IMM BASED KALMAN FILTERS}

The principle of IMM based Kalman filters algorithm can be summarized as follows: The trajectory of moving object is filtered with Kalman filters using a number of different models (maneuvering model or different parameter nonmaneuvering models). At the beginning and the end of filtering, weighted sums of the results of each model are computed according to their probability. Assuming that there are $r$ motion models ( $r$ state transition equations):

$$
X_{j}(k+1)=\Phi_{j}(k) X_{j}(k)+G_{j}(k) W_{j}(k) j=1,2, \ldots r
$$

where the mean is zero, covariance matrix is white noise sequence. And measurement model is:

$$
Z_{j}(k)=H_{j}(k) X_{j}(k)+V_{j}(k)
$$

Transition probability from model $i$ to model $j$ is $p_{i j}$, the probability of model $j$ at time $k-1$ is $\mu_{j}(k-1)$, prediction probability of model j(normalization constant) is $\bar{c}_{j}=\sum_{i=1}^{r} p_{i j} \mu_{i}(k-1)$, the mixed probability from model $i$ to model $j: \mu_{i j}(k-1 / k-1)=\sum_{i=1}^{r} p_{i j} \mu_{i}(k-1) / \bar{c}_{j}$. The mixed state estimation of model $j$ :

$$
\hat{X}_{0 j}(k-1 / k-1)=\sum_{i=1}^{r} \hat{X}_{i}(k-1 / k-1) \mu_{i j}(k-1 / k-1)
$$

The mixed variance estimation of model $j$ : 


$$
\begin{aligned}
& P_{0 j}(k-1 / k-1)= \\
& \sum_{i=1}^{r} \mu_{i j}(k-1 / k-1)\left(P_{i}(k-1 / k-1)+\tilde{X}_{\Delta}(k-1 / k-1)\left[\tilde{X}_{\Delta}(k-1 / k-1)\right]^{T}\right)^{(10)}
\end{aligned}
$$

With $\tilde{X}_{\Delta}(k-1 / k-1)=\hat{X}_{i}(k-1 / k-1)-\hat{X}_{0 j}(k-1 / k-1)$.

Updating model probability: The likelihood functions of model $j$ :

$$
\Lambda_{j}(k)=\frac{1}{(2 \pi)^{n / 2}\left|S_{j}(k)\right|^{1 / 2}} \exp \left\{-\frac{1}{2} v_{j}^{T} S_{j}^{-1}(k) v_{j}\right\}
$$

With $v_{j}(k)=Z(k)-H(k) \hat{X}_{j}(k / k-1)$ is the innovation $S_{j}(k)=H(k) P_{j}(k / k-1) H(k)^{T}+R(k)$ is the variance of the innovation. The probability of model $j$ is $\mu_{j}(k)=\Lambda_{j}(k) \bar{c}_{j} / c$.

Interactive Output: The total state estimation:

$$
\hat{X}(k / k)=\sum_{j=1}^{r} \hat{X}_{j}(k / k) \mu_{j}(k)
$$

The total estimation of measurement error variances:

$$
P_{0 j}(k / k)=\sum_{j=1}^{r} \mu_{j}(k / k)\left(P_{i}(k / k)+\tilde{X}_{\delta}(k / k)\left[\tilde{X}_{\delta}(k / k)\right]^{T}\right)(13)
$$

With $\tilde{X}_{\delta}(k / k)=\hat{X}_{j}(k / k)-\hat{X}(k / k)$.

We use three models in this paper, the 1st model is nonmaneuvering model, the system noise variance of the $1 \mathrm{st}$ model is zero; the 2nd and the 3rd model are maneuvering model. Assume that the system noise variance of the 2 nd model is $Q=0.001 \mathrm{I}$, and the system noise variance of the $3 \mathrm{rd}$ model is $Q=0.0144 \mathrm{I}$. Markov transition probability matrix of control model is expressed as follow:

$$
p=\left[\begin{array}{ccc}
0.95 & 0.025 & 0.025 \\
0.025 & 0.95 & 0.025 \\
0.025 & 0.025 & 0.95
\end{array}\right]
$$

Assuming that the transition probabilities of the models are $\mu_{1}=0.8, \mu_{2}=0.1, \mu_{3}=0.1$ (These parameters can be modified). The parameters of these models are defined as follow:

$$
x_{1}=\left[\begin{array}{c}
x \\
\dot{x} \\
y \\
\dot{y} \\
0 \\
0
\end{array}\right], x_{2}=x_{3}=\left[\begin{array}{c}
x \\
\dot{x} \\
y \\
\dot{y} \\
\ddot{x} \\
\ddot{y}
\end{array}\right]
$$

$$
G_{1}=\left[\begin{array}{cc}
T^{2} / 2 & 0 \\
T & 0 \\
0 & T^{2} / 2 \\
0 & T \\
0 & 0 \\
0 & 0
\end{array}\right], G_{2}=G_{3}=\left[\begin{array}{cc}
T^{2} / 4 & 0 \\
T / 2 & 0 \\
0 & T^{2} / 4 \\
0 & T \\
1 & 0 \\
0 & 1
\end{array}\right],
$$

$$
\begin{gathered}
\Phi_{1}=\left[\begin{array}{llllll}
1 & T & 0 & 0 & 0 & 0 \\
0 & 1 & 0 & 0 & 0 & 0 \\
0 & 0 & 1 & T & 0 & 0 \\
0 & 0 & 0 & 1 & 0 & 0 \\
0 & 0 & 0 & 0 & 0 & 0 \\
0 & 0 & 0 & 0 & 0 & 0
\end{array}\right], \\
\Phi_{2}=\Phi_{3}=\left[\begin{array}{lllllc}
1 & T & 0 & 0 & T^{2} / 2 & 0 \\
0 & 1 & 0 & 0 & T & 0 \\
0 & 0 & 1 & T & 0 & T^{2} / 2 \\
0 & 0 & 0 & 1 & 0 & T \\
0 & 0 & 0 & 0 & 1 & 0 \\
0 & 0 & 0 & 0 & 0 & 1
\end{array}\right]
\end{gathered}
$$

Initial state would be expressed as:

$\hat{X}(2 / 2)=\left[\begin{array}{lllll}z_{x}(2) \frac{z_{x}(2)-z_{x}(1)}{T} & z_{y}(2) \frac{z_{y}(2)-z_{y}(1)}{T} & 0 & 0\end{array}\right]$

$$
p(2 / 2)=\left[\begin{array}{cccccc}
\delta_{x}^{2} & \delta_{x}^{2} / T & 0 & 0 & 0 & 0 \\
\delta_{x}^{2} / T & 2 \delta_{x}^{2} / T^{2} & 0 & 0 & 0 & 0 \\
0 & 0 & \delta_{y}^{2} & \delta_{y}^{2} / T & 0 & 0 \\
0 & 0 & \delta_{y}^{2} / T & 2 \delta_{x}^{2} / T^{2} & 0 & 0 \\
0 & 0 & 0 & 0 & 0 & 0 \\
0 & 0 & 0 & 0 & 0 & 0
\end{array}\right]
$$

The mean value of filtering error is:

$$
\bar{e}_{x}(k)=\frac{1}{M} \sum_{i=1}^{M}\left[x_{i}(k)-\hat{x}_{i}(k / k)\right]
$$

The standard deviation of filtering error is:

$$
\sigma_{\hat{x}}=\sqrt{\frac{1}{M} \sum_{i=1}^{M}\left[x_{i}(k)-\hat{x}_{i}(k / k)\right]^{2}-\left[\bar{e}_{x}(k)\right]^{2}}
$$

where $M$ denotes simulation number of Mente-carlo, $k=1,2, \ldots, N, N$, is the sampling number.

\section{Simulation}

In this section, the maneuvering target tracking performance of EBPSK modulated JRC system with IMM based Kalman filter algorithm is simulated. Simulated orbit: at the beginning, the target bears a constant velocity on y-axis, 
and its initial value is $450 \mathrm{~m} / \mathrm{s}$. The target moves in uniformly accelerated motion and makes a 90-degree sharp turn on $\mathrm{x}$-axis between 200th-300th scanning intervals, accelerated velocity is $u_{x}=u_{y}=20 \mathrm{~m} / \mathrm{s}^{2}$. Then the target makes a gradual 90-degree turn again on $\mathrm{x}$-axis between 305 th-330th scanning intervals, accelerated velocity is $u_{x}=u_{y}=3 \mathrm{~m} / \mathrm{s}^{2}$.

At the last, the target is moving in uniform motion again between 330th-450th scanning intervals. In Table I, a summary of the important parameters of the simulation model is provided.

\section{TABLE I: SIMULATION PARAMETER}

\begin{tabular}{ll}
\hline \hline Simulation parameter & \\
\hline Carrier frequency: & $f_{1}=10 \mathrm{GHz}$ \\
Down-conversion & $100 \mathrm{MHz}$ \\
Sampling frequency & $f_{1}=1 \mathrm{GHz}$ \\
Modulation parameter & $K=2 ; N=7$ \\
scanning period & 100 and 200 \\
Standard deviation of the noise & \\
\hline \hline
\end{tabular}

Real tracks and filtering tracks of flight target corresponding to standard deviation of the noise 100 and 200 are shown in Fig. 2. The simulations are in the AWGN channel. The results are obtained from 500 Monte Carlo simulations.

The shape of filter curve can be adjusted and swings slightly at the two sides of true target track. And the margin fluctuation of filter curve get higher as increasing the standard deviation of noise as shown in Fig. 2, respectively.

The experiments showed the EBPSK modulated JRC system achieved good filter effect and could be effective to tract the position of the target in some cases, whether flight target is in maneuvering or non-maneuvering.

Maneuvering target is measured on $\mathrm{x}$ and $\mathrm{y}$-axis, respectively. The filtering error mean curves of the flight target corresponding to standard deviation of the noise 100 and 200 are shown in Fig. 3.

The mean curves of filter values on $\mathrm{x}$ and $\mathrm{y}$ axis are show in Fig. 3. There are 4 major fluctuations on $x$ and $y$-axis for the mean curves due to two sharp turning. The fluctuations of the means curves filter value would become small when the flight target travels in straight lines.

The filtering error standard deviation cruves of the flight target corresponding to standard deviation of the noise 100 and 200 are shown in Fig. 4.

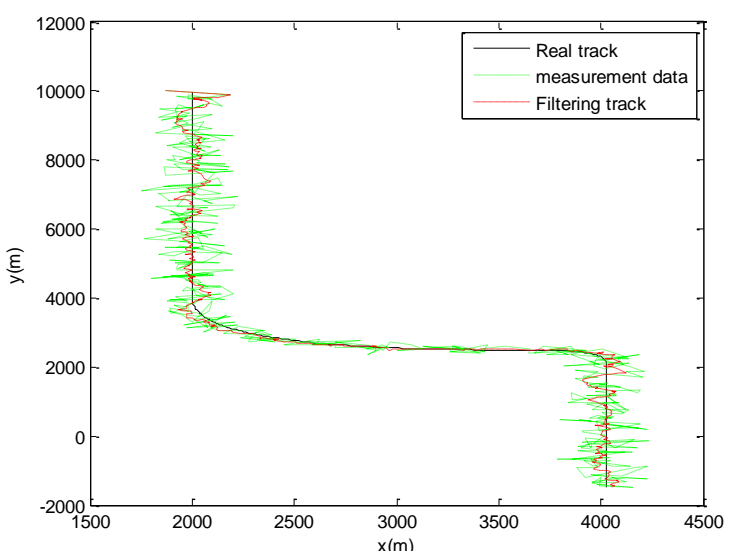

(a)

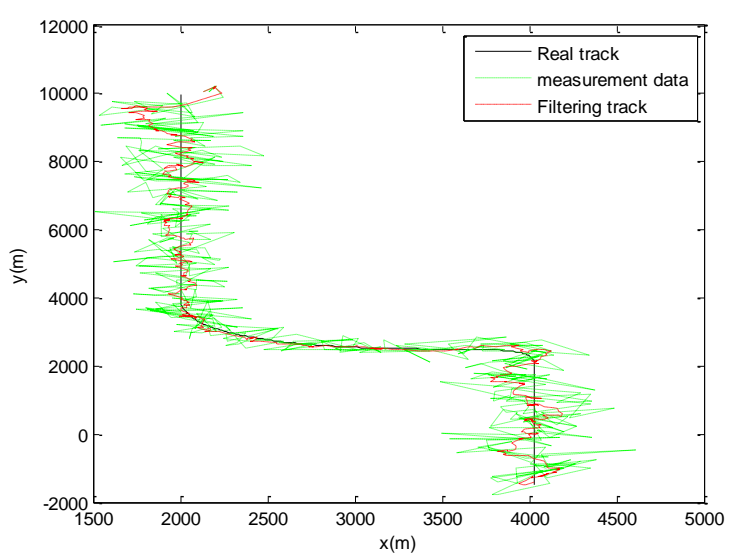

(b)

Fig. 2. Real tracks and filtering tracks corresponding to standard deviation of the noise 100 and 200 .
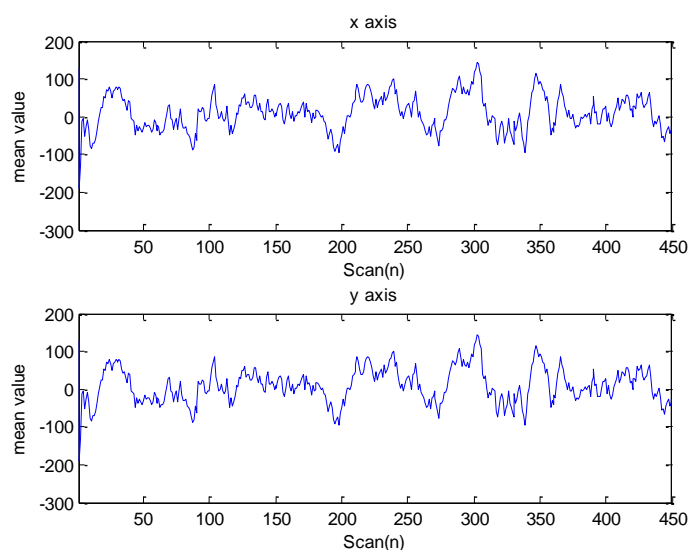

(a)
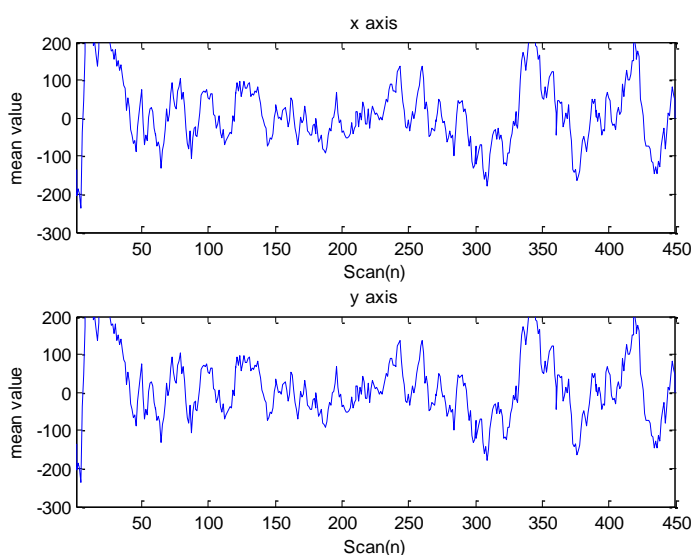

(b)

Fig. 3. The filtering error mean corresponding to standard deviation of the noise 100 and 200
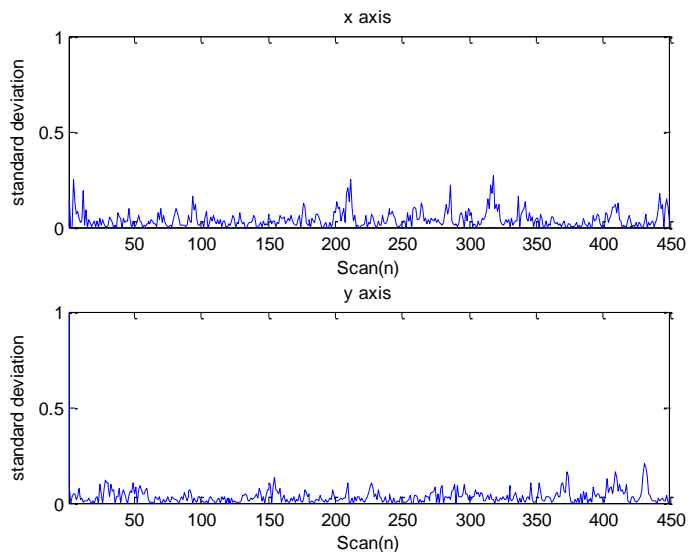

(a) 

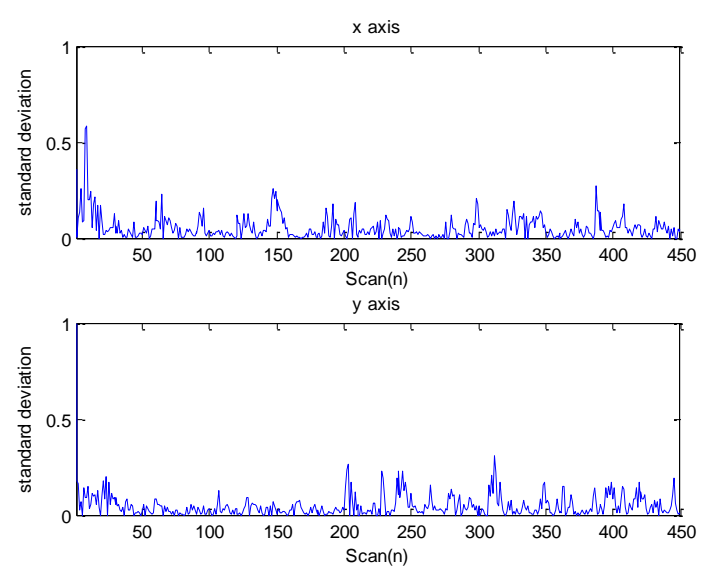

(b)

Fig. 4. The standard deviation corresponding to standard deviation of the noise 100 and 200

The standard deviation curves of filter values on $\mathrm{x}$ and $\mathrm{y}$ axis are show in Fig. 4. The target motion process can be shown clearly to distinguish between Uniform and maneuvering. There are two major fluctuations on $\mathrm{x}$ and $y$-axis for the standard deviation curves due to two sharp turning. The former is bigger than the latter because the first turning is sharper than the second turning. The standard deviation of filter value would become really small after the filtering process get into the steadiness.

From the above analysis we could see, The EBPSK modulated JRC system based on IMM Kalman filters would be less affected by the maneuvering of the target. The proposed system has the capability of fast and precise maneuvering target tracking, which costs a large computation.

\section{SOME COMMON MistaKeS}

The tracking and estimation problems of maneuvering target are considered, and an EBPSK modulated JRC working scheme with Kalman filters is proposed in this paper. The maneuvering tracking model of the proposed system is given. The generalized likelihood ratio (GLR) and marginalized likelihood ratio (MLR) method are proposed for maneuvering target tracking. Weighted least square estimation for target location and Kalman filtering for tracking based on one or more different models are used in the proposed system. Furthermore, the adaptive maneuvering target tracking algorithm using an IMM based Kalman filters is proposed. The simulations and measurement results of the proposed system demonstrate excellent tracking performance for maneuvering targets.

\section{REFERENCES}

[1] Y. Yao and L. N. Wu, "Performance analysis of single-frequency MPPSK integrated system for ranging applications," The Scientific World Journal, 2014.

[2] Y. Yao and L. N. Wu, "Studies of a dual frequency EBSPK-MODEM based radar communication transceiver," Journal of Electronics and Information Technology, vol. 36, no. 8, pp. 1786-1791, 2014.

[3] C. Sturm and W. Wiesbeck, "Waveform design and signal processing aspects for fusion of wireless communications and radar sensing," Proceedings of the IEEE, vol. 99, no. 7, pp. 1236-1259, 2001.
[4] D. W. Winters, "Target motion and high range resolution profile generation," IEEE Transactions on Aerospace and Electronic Systems, vol. 48, no. 3, pp. 2140-2153, 2012.

[5] L. N. Wu and M. Feng, "On BER Performance of EBPSK-MODEM in AWGN channel," Sensors, vol. 10, no. 4, pp. 3824-3834, 2010.

[6] C. Xianqing and W. Lenan, "A novel detection scheme for EBPSK system," Mathematical Problems in Engineering, pp. 1-14, 2012.

[7] M. Feng, L. N. Wu, and P. Gao, "From special analogous crystal filters to digital impacting filters," Digital Signal Processing, vol. 22, no. 4, pp. 690-696, 2012

[8] J. Sun, W. Fang, and W. Xu, "A quantum-behaved particle swarm optimization with diversity-guided mutation for the design of two-dimensional IIR digital filters," IEEE Transactions on Circuits and Systems II: Express Briefs, vol. 57, no. 2, pp. 141-145, 2010.

[9] M. Feng, L. N. Wu, D. Jiajia, and Q. Chenhao, "BER analysis and verification of EBPSK system in AWGN channel," IEICE Transactions on Communications, vol. E94-B(3), pp. 806-809.

[10] T. Ho and B. Chen, "Novel extended Viterbi-based multiple model algorithms for state estimation of discrete-time systems with Markovjump parameters," IEEE Transactions on Signal Processing, vol. 54, no. 2, pp. 393-404, 2006.

[11] Z. Zhong, H. Meng, and X. Wang, "Extended target tracking using an IMM based Rao-Blackwellised unscented Kalman filter," in Proc. 2008 9th International Conference on ICSP, 2008, pp. 2409-2412.

[12] Y, Kirubarajan and T, X. Lin, "Probabilistic data association techniques for target tracking with applications to sonar, radar and EO sensors," IEEE, Aerospace and Electronic Systems Magazine, vol. 20, no. 8, pp. 37-56, 2005.

[13] M. Xing, J. Su, G. Wang, et al. "New parameter estimation and detection algorithm for high speed small target," IEEE Transactions on Aerospace and Electronic Systems, vol. 47, no. 1, pp. 214-224, 2011.

[14] X. Chen, J. Guan, Y. He, et al. "Detection of low observable moving target in sea clutter via fractal characteristics in fractional Fourier transform domain," IET Radar, Sonar \& Navigation, vol. 7, no. 6, pp. 635-651, 2013.

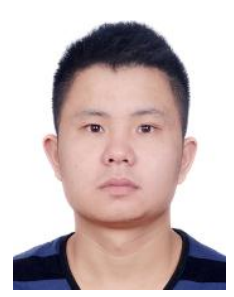

Yu Yao was born in Jiangxi, China in 1986. He has received the B.E. degree from the School of Information Science and Engineering, Nanchang University, China, in 2007. He is currently pursuing his $\mathrm{PhD}$ degree in the School of Information Science and Engineering, Southeast University, China, under the supervision of Prof. Lenan Wu. His research interests include the communication and radar signal processing, particularly with the radar and communication system integration.

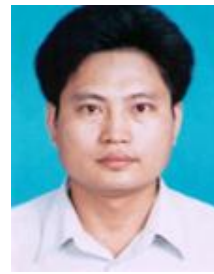

Huanglong Teng is a senior engineer in China Electronics Technology Group Corporation No.7 Research Institute. He finished his bachelor's degree in Beijing Institute of Technology and master's degree in South China University of Technology. He has engaged in mobile communication for more than 20 years. His main research areas include wireless sensor network, internet of things.

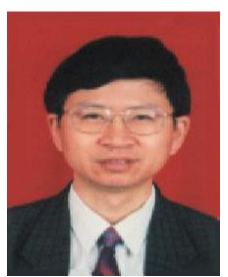

Lenan Wu received his M.S. degree in electronics communication system from Nanjing University of Aeronautics and Astronautics, China, in 1987, and $\mathrm{Ph} . \mathrm{D}$. degree in signal and information processing from Southeast University, China, in 1997. Since 1997, he has been with Southeast University, where he is a professor, and the director of the Multimedia Technical Research Institute. He is the author or coauthor of over 400 technical papers and 11 textbooks, and is the holder of 20 Chinese patents and 1 International patent. His research interests are multimedia information systems and communication signal processing. 